\title{
Labial Fusion Causing Coital and Voiding Difficulty in a Young Woman
}

\author{
Murat Ozekinci $^{1}$, Selcuk Yucel ${ }^{2}$, Cem Sanhal $^{1}$, Munire Erman Akar ${ }^{1}$ \\ ${ }^{1}$ Department of Obstetrics and Gynecology, Akdeniz University School of Medicine, Antalya, Turkey \\ ${ }^{2}$ Department of Urology, Akdeniz University School of Medicine, Antalya, Turkey \\ Email: mnirea@yahoo.com
}

Received November 6, 2012; revised December 15, 2012; accepted December 29, 2012

\begin{abstract}
Adhesions of the labia are extremely rare in the reproductive population with only a few cases described in the literature. We report a young female patient with coital and voiding difficulty. The patient was a 22-year-old woman presented with voiding difficulty and inability to achieve sexual intercourse. Clinical examination revealed normally developed labia majora, clitoris, and vaginal opening, but fused labia minora and pinhole external urethral orifice. Intraoperatively, labial fusion was dissected with sharp dissection. Urethral dilatation was performed with dittel dilators. Local estrogen ointment was administered postoperatively for four weeks resulting with complete resolution of coital and voiding symptoms.
\end{abstract}

Keywords: Labial Fusion; Labial Adhesion

\section{Introduction}

Labial fusion (LF) is defined as partial or complete adhesion of the labia minora and estimated to occur in $0.6 \%$ $5 \%$ of prepubertal girls [1]. But this condition is a rare clinical entity in adults, only a few cases have been reported in the literature and these cases occurred predominantly in post-menopausal women rather than in reproductive ages [1,2]. Although majority of the reported cases are aymptomatic, rarely they may present with post-void dripping (urinary incontinence), urinary tract infections, vaginitis (vaginal irritation), hematuria and urinary frequency [1]. Vaginal inflammation-irritation, hypoestrogenism, local trauma, lack of sexual activity, female circumcision, vaginal lacerations in childbirth and recurrent urinary tract infections are formerly reported as the contrubuting factors for LF in adults [2]. We describe a case of near-complete labial fusion in a young woman presenting with coital and voiding difficulty, its management and literature review.

\section{Case Report}

A 22-year-old woman was admitted to our gynecology department with the complaint of unsuccessful vaginal coital activity attempt. She had a history of regular menstrual cycles since menarche (at the age of 14 years). Her personal medical history was unremarkable except for urinary symptoms. She described nocturia, retention due to voiding difficulty and two episodes of urinary tract infection in the last two years. She had no history of any trauma or sexual assault in any time as well. Gynecological examination revealed partial adhesion of the labia minora (Figure 1). Labia majora were natural in shape and a pinhole urethral orifice could be seen. Urine analysis and urine culture were negative for infection. Hormonal analysis revealed normal estrogen and gonadotrophin levels. Pelvic ultrasonography disclosed an antevert uterus and normal appearing adnexia. Abdominopelvic MRI affirmed normal urinary system.

Under general anesthesia, in lithotomy position, inspection of the external genitelia revealed fused labia minora and external urethral orifice. Urethral catheterizetion could only be done with $10 \mathrm{~F}$ silicon catheter. The patient was intraoperatively consulted with urology. Afterwards dilatation of urethra had been performed with dittel dilatators till number 32. Labial adhesions were released by sharp dissection and the released edges were sutured (Figure 2). Intraoperative visualization confirmed that cervix and vagina were anatomically normal in shape and colour. Vaginal pack containing fucidic acid was applied for 24 hours postoperatively. The patient was discharged with daily topical estrogen ointment therapy to be applied for four weeks. On her postoperative control at the end of six weeks, labial tissues were found to be completely healed (Figure 3). She stated that she had no pain during intercourse postoperatively and her 


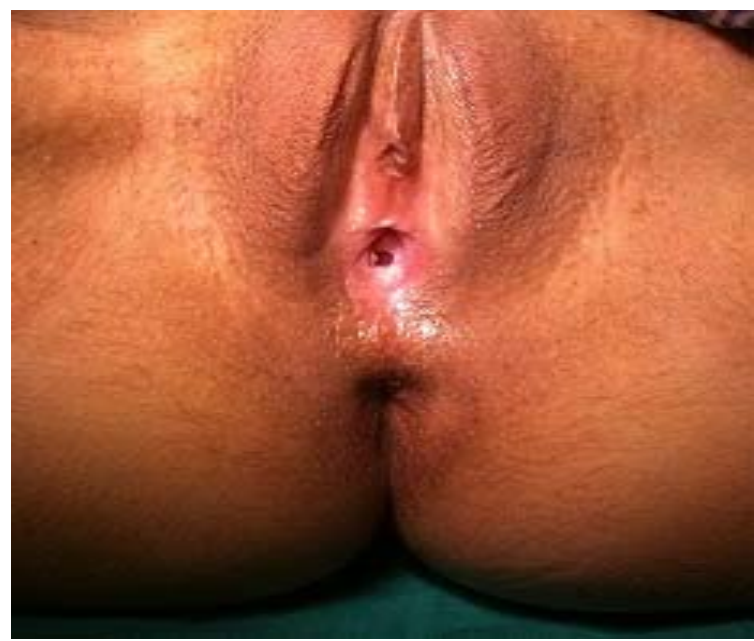

Figure 1. Preoperative view of the labial fusion defect.

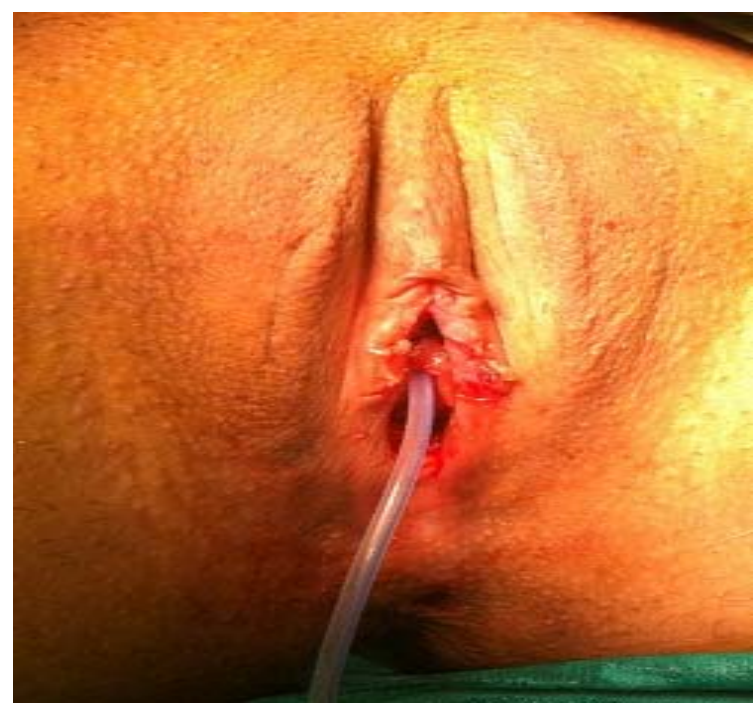

Figure 2. Early postoperative view of the case following urethral dilatation and catheterization.

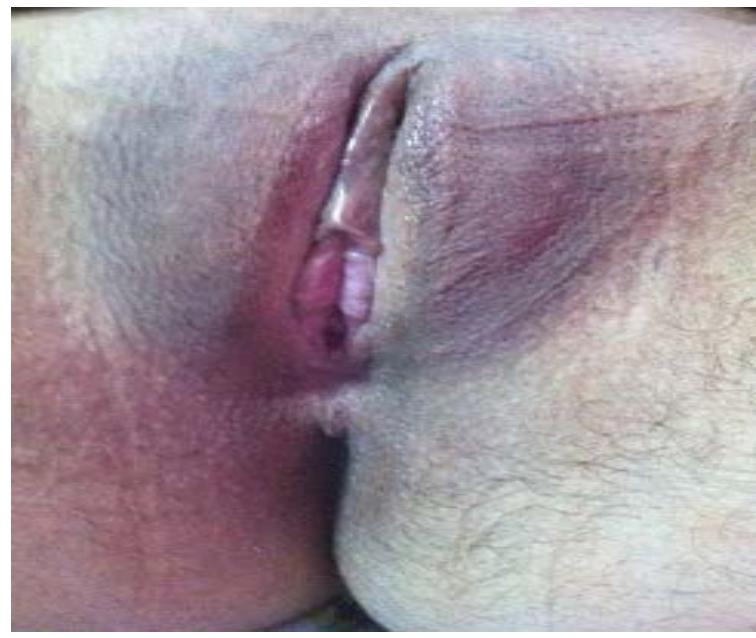

Figure 3. Postoperative view of the case at postoperative six weeks. voiding problems had resolved.

\section{Discussion}

Labial fusion (LF), a state of partial or complete adhesion of the labia minora, generally occur in children or post-menopausal women, but is extremely rare in reproductive ages [3]. The relative hypoestrogenic enviroment is suggested as the main predisposing factor of this disorder in prepubartal girls [1]. Sexual abuse or genital trauma has also been implicated as an other major causative factor in various reports in older girls [4]. In adults including postmenopausal women, LF is more associated with recurrent urinary tract infections, vulvovaginitis, genital trauma, hypoestrogenism and lack of sexual activity [5]. Most predominant accompanying symptoms of LF in postmenopause are vulvar soreness, pruritis and urinary symptoms such as dysuria, urinary incontinenceretention and voiding difficulties.

We can exclude hypoestrogenemia as the cause of her LF in our patient with a history of regular menses, normal ovaries at ultrasound and normal gonadotrophin values. Also, she did not assert a history of genital trauma or sexual abuse which was formerly reported as a possible cause of LF in the reproductive age group [6].

Our patient presented with nocturia, urinary retention due to voiding difficulties and urinary tract infection history probably as a result of LF.

Topical estrogen cream is generally accepted as the initial therapy for superficial labial adhesions which may reduce the irritation of the vaginal mucous membranes and ease subsequent repair particularly in prepubertal girls and postmenopausal women [2,7]. One study comparing topical estrogen and betametasone treatment for the treatment of LF in prepubertal girls noted that betametasone therapy was superior to topical estrogen therapy in terms of efficacy, recurrence and side effects [1]. Surgery should become the first option for treatment, in patients with complete, thick adhesions and a long history of LF. Our patient's age, status and the necessity of expeditious treatment made us choose surgery instead of topical therapy. With the concomitant use of topical estrogen therapy, successful vaginal intercourse were achieved at 4 th postoperative week.

Although it is a rare event, LF should be considered in the differential diagnosis of the patients presenting with sexual dysfunction.

\section{REFERENCES}

[1] L. Mayaoglu, L. Dulabon, M. N. Alguacil, D. Pfaff and J. Schober, "Success of Treatment Modalities for Labial Fusion: A Retrospective Evaluation of Topical and Surgical Treatments," Journal of Pediatric and Adolescent Gynecology, Vol. 22, No. 4, 2009, pp. 247-253. 
doi:10.1016/j.jpag.2008.09.003

[2] A. Dirim and E. Hasirci, "Labial Fusion Causing Urinary Incontinance and Recurrent Urinary Tract Infection in a Postmenopausal Female: A Case Report,” International Urogynecology Journal, Vol. 22, No. 1, 2011, pp. 119120. doi:10.1007/s00192-010-1205-2

[3] Y. Tsujita, J. Asakuma, T. Kanbara, T. Yoshii, R. Azuma, M. Sumitomo and T. Asano, "A Case of Labial Adhesion in a Reproductive Woman,” Hinyokika Kiyo, Vol. 56, No. 8, 2010, pp. 463-465.

[4] C. D. Berkowitz, S. L. Elvik and M. K. Logan, "Labial Fusion in Prepubescent Girls: A Marker for Sexual Abuse?” American Journal of Obstetrics \& Gynecology, Vol. 156, No. 1, 1987, pp. 16-20.
[5] B. Lambert, "Complete Adult Vulvar Fusion: A Case Report,” Journal of Obstetrics and Gynaecology Canada, Vol. 26, No. 5, 2004, pp. 501-502.

[6] R. K. Kumar, A. Sonika, C. Charu, K. Sunesh and M. Neena, "Labial Adhesions in Pubertal Girls," Archives of Gynecology and Obstetrics, Vol. 273, No. 4, 2006, pp. 243-245. doi:10.1007/s00404-005-0060-8

[7] H. Muppala and A. Meskhi, "Voiding Dysfunction Due to Longstanding Labial Fusion in an Elderly Woman: A Case Report,” International Urogynecology Journal and Pelvic Floor Dysfunction, Vol. 20, No. 2, 2009, pp. 251252. 